\title{
On the research of cultural relic restoration under reverse design
}

\author{
Chao Zhang ${ }^{1}$, Ruikun $\mathrm{Li}^{2, *}$, Wenhui Chen ${ }^{3}$, Xupeng Wang ${ }^{4}$ \\ ${ }^{1}$ School of Automation and Information Engineering, Xi'an University of Technology, Xi' an 710048, China \\ ${ }^{2}$ Department of Industrial Design, Xi'an University of Technology, Xi'an, 710054, China \\ ${ }^{3}$ Department of Industrial Design, Xi'an University of Technology, Xi'an, 710054, China \\ ${ }^{4}$ Department of Industrial Design, Xi'an University of Technology, Xi'an, 710054, China
}

\begin{abstract}
At present, artificial restoration of cultural relics has been unable to solve the difficulties in restoration and protection of cultural relics, as well as low restoration rate a series of problems.In order to solve the above problems, this article conducts in-depth research on the emerging computer technology that can be used for cultural relic restoration, and proposes a computer-aided cultural relic restoration and reconstruction process based on the reverse design method, and explains the application of 3D printing technology to the restoration of ceramic cultural relics. In this paper, the unearthed cultural relics are protected to the maximum extent research, which provides a reference for improving the scientific nature and durability of cultural relics protection and inheritance.
\end{abstract}

\section{Introduction}

As witnesses of history, cultural relics have witnessed the rapid development and changes of the country and the nation. However, the excavation of modern cultural relics is confronted with various problems, such as the irreversible natural deterioration phenomena such as fading, discoloration, yellowing, deformation and rupture caused by improper protection of unearthed cultural relics and natural environment[1]. These effects are difficult to restore or repair, only through modern new technology to better preserve and repair, in order to maximize the protection of the integrity of cultural relics.

The restoration of cultural relics can be divided into the preservation and re-creation of cultural relics, covering many aspects of knowledge and being highly comprehensive.Reverse engineering refers to already owning an object, mastering the surface shape, space size, and internal components of the object, and then printing some of its small parts.Reverse seeking can adjust the placement or structure of small parts, make certain changes, and produce creative designs. In the reverse process, if there is no change, the production based on the original unchanged is the most basic imitation.Reverse engineering technology is an important technical means for digesting and absorbing advanced technology to develop, design and innovate new products.Therefore, while making use of existing technologies, we should give full play to the powerful creativity of human beings, so that cultural relics are no longer the product of one era, but the crystallization of multiple eras, containing the cultural connotations of people of different eras, showing a richer spirit of The Times, and laying a more solid cultural foundation of Our country.

The introduction of modern design and manufacturing technology will combine the restoration of cultural relics with modern computers and manufacturing technologies, forming a new technological growth point [2].On the one hand, they avoid the single boring and repetitive high-precision restoration work, create a more comfortable restoration environment and reduce restoration costs; on the other hand, it can also improve the effect of cultural relic restoration, such as more uniform painting, more symmetrical shape, and surface Smoother, etc., reduces the secondary damage to the cultural relics, and achieves the effect that cannot be achieved by manual repair.

\section{Computer technology for restoration of cultural relics}

The new computer technology used for cultural relic restoration is based on the data extraction method of incomplete boundary features, the fuzzy recognition and identification technology of cultural relic residues, computer three-dimensional graphics mutation processing technology, generating cultural relic restoration models[3], making cultural relic restoration

\footnotetext{
*Corresponding author's e-mail: 1064068962@qq.com
} 
prostheses, and completing cultural relics Overall repair, this type of technology can reduce the time used by the repairer, save repair costs, and improve repair quality. The current mature technologies are as follows:

\subsection{D laser scanning technology}

Three-dimensional laser scanning technology is another new surveying and mapping technology following the spatial positioning of the Global Positioning System in the 1990s.Compared with traditional technology, it can obtain information accurately and quickly. There is no need to touch the target cultural relics in the process of obtaining information. The principle is to quickly collect the three-dimensional coordinates of the surface of the measurement object through high-speed laser scanning measurement with large area and high resolution. To obtain the spatial information of the object, which provides a new method for the rapid establishment of the target three-dimensional model [4].

RENISHAW has proposed an optical sampling head (OP3M optical sampling head) that can be designed for laser data collection. It has a very fast collection speed [5,6], and the probe will not come into contact with the sample, so It is easier to collect loose and soft materials, such as measuring the contours of the surface that are not conducive to contact, such as sharp corners and recesses.

\subsection{Structured light method}

The structured light method is to irradiate a certain pattern of light on the surface of the sample to be tested, and then capture the image of the reflected light, and obtain the position of the point on the surface of the sample by comparing the differences between the different modes [7].Its advantages are low cost, no need for precision equipment such as coordinate measuring machines, relatively low cost, but not high accuracy, and relatively complicated operation.

\subsection{Three-dimensional simulation repair technology}

Three-dimensional simulation repair technology is mainly used to create three-dimensional models of defective parts of cultural relics. There are many 3D design software nowadays, which can be roughly divided into small and large. One is small three-dimensional design software. The biggest feature of these software is simple and easy to get started. The disadvantage is that the function is single and other features cannot be guaranteed.The other is large-scale 3D design software, such as 3DS VIZ, SoftImage, AutoCAD, MAYA, 3D Studio MAX, etc. $[8,9]$.These softwares are extremely powerful, especially in terms of modeling and rendering functions, and can reach the level of real shooting.

\subsection{Digital image repair technology}

This technology is based on the existing image information to repair the damaged part, as shown in Figure 1. This technology makes the restored image look like the real effect of the original, which is the so-called high-precision simulation.However, digital image restoration is a typical ill-conditioned problem. It is difficult for the restoration algorithm to efficiently self-feed back the restoration results, which leads to the general problems of poor robustness and low automation of the algorithm. There is still a certain distance from the real practicality [11].

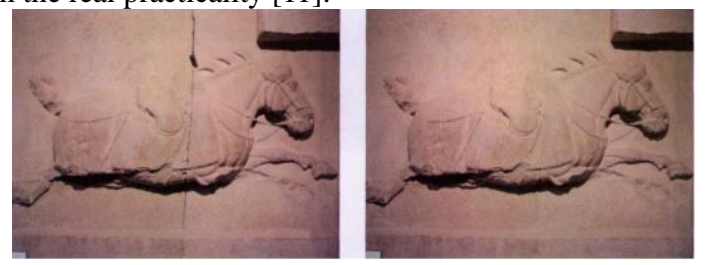

Fig.1. Comparison before and after the virtual repair of cracks using the variational PDE repair model

\section{Reconstruction process of cultural relics restoration}

Three-dimensional measurement technology, fuzzy control theory, graphic mutation technology, and finite element structure analysis technology are used for cultural relic restoration design, and $3 \mathrm{D}$ printing technology is used to achieve complete restoration of cultural relics [12,13]. For cultural relics restoration workers, these technologies are of great significance in protecting the restoration of special cultural relics with fragile materials and precious value. The repair process is shown in Fig.2.

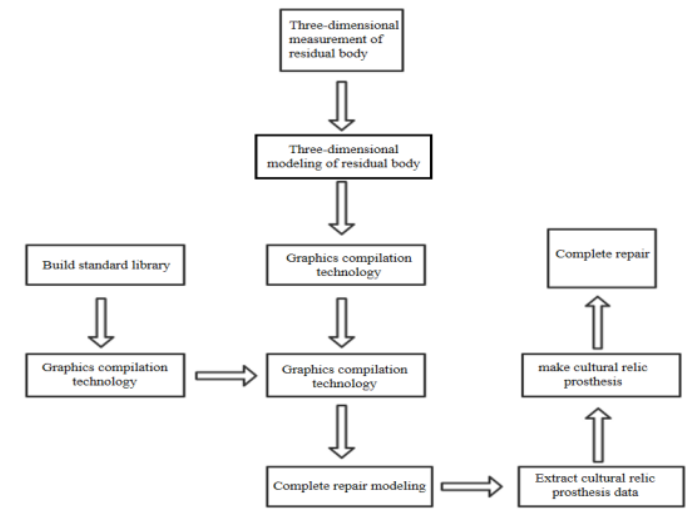

Fig.2. Computer-aided cultural relic restoration flowchart

This process is based on the reverse design method for the restoration and reconstruction of cultural relics. First of all, the use of incomplete cultural relic entities to perform three-dimensional imaging scans can record the approximate size and virtual shape of the debris in the computer.Secondly, the basic shape of the remnant is sketched in the computer. Since cultural relics of different dynasties have similar shapes, fuzzy matching is carried out in the cultural relic standard database after obtaining the dynasty to which the remnant belongs.This step is to perform virtual filling of the missing parts of the residual body, perform fitting modeling of the missing parts that meet the general requirements, and match the model with the residual cultural relics.Finally, the relatively complete cultural relics are 3D printed out for secondary correction, and the integrity of the shape is continuously corrected, and the restoration of the cultural relics is finally completed. Greatly reduce the repair time and repair resources in the work.

\section{Application of 3D printing technology in repairing ceramic relics}

At present, most of the splicing of fragments in the process of cultural relic restoration is still manual splicing. Due to the large number of cultural relics fragments, the splicing 
relationship between the fragments is difficult to determine, and some cultural relics fragments are large in size and heavy, and they are not easy to move during the splicing attempt. The repair brought difficulties.Using 3D printing technology to splice cultural relics can effectively overcome the above-mentioned problems. First, a three-dimensional laser scanner is used to obtain a three-dimensional digital model of the cultural relic fragments, and then a $3 \mathrm{D}$ printing device is used to generate a solid model of each fragment. Finally, complete the splicing restoration of the actual cultural relics according to the determined splicing relationship.

Take a ceramic jar cultural relic restoration as an example, the specific process is shown in Fig.3, The effect comparison before and after repair is shown in Fig.4.

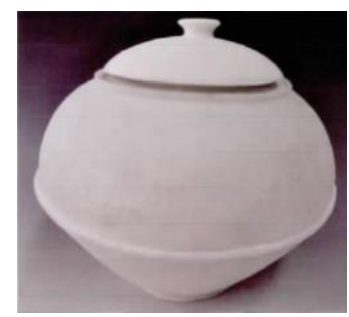

(a)3D printed pottery

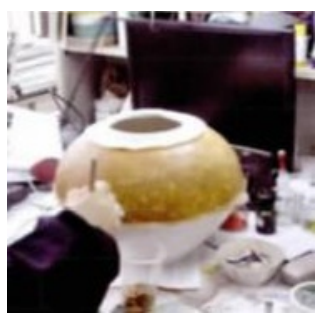

(b)Apply a layer of blended loess

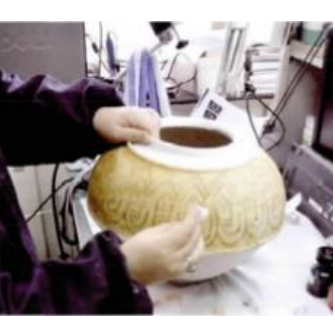

(c) Wipe off the surface half dry background

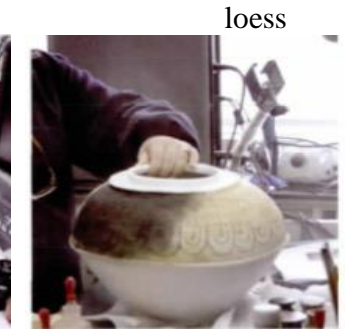

(d) Inkjet on black when it is

Fig.3. Flow chart for restoration of clay pot cultural relics
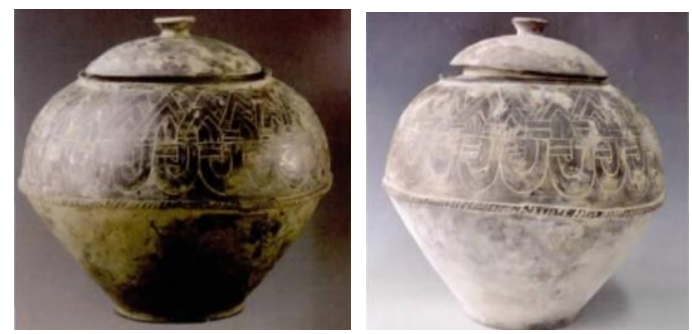

(a)Original cultural relics (b) Restored cultural relics

Fig.4. Contrast before and after restoration of cultural relics

3D printing can collect complete and true information about the three-dimensional space, color and size of cultural relics without touching the cultural relics, which is of epoch-making significance. Moreover, for symmetrical objects, multiple manual shaping operations can be omitted, and it can be completed only by using the principle of mirroring, and the accuracy of cultural relics can also be guaranteed.

\section{Conclusion}

Based on the reverse design of ancient cultural relics repair by modern science and technology, can the damage of cultural relics under the condition of without touching for more more complete files, establish mathematical model for cultural relics original real information electronic information database, reduce the cost of restoration of improving the quality of the restoration, at the same time for subsequent excavation and restoration of cultural relics provides more scientific and effective method and basis, has a great development space and development prospects, is worth our further research.

\section{References}

1. Li Jingsheng. Discussion and practical application of cultural relic restoration concepts[J]. Chinese Cultural Relics Science Research(2012).

2. Li Dichen; Qiu Zhihui; Ning Juntao; Lu Bingheng; Ma Tao; Wang Zhan. Computer-aided Cultural Relics Restoration Design and Rapid Restoration Methods[J]."The Second Annual Conference of China Association for the Protection of Cultural Relics" (2002).

3. Zhao Lisha. Research on three-dimensional restoration of French objects based on double mirror reflection [J], "Master Thesis of Hebei University of Technology" (2011).

4. Yao Meng, Zhang Ling, Abdulaziz, Liu Xiao. Three-dimensional reconstruction, rendering and representation of ancient cultural relics based on modeling[J].East China Normal University,Department of Electronic Science and Technology (2000).

5. Adam J. A. Probing beneath the sea [J]. IEEE Spectrum (S0018-9235), 22(4): 55-64(1985),.

6. Yongjie Pang, You Shang. Software Design Techniques for the Man-Machine Interface to Autonomous Underwater Vehicles [C]//Proc. of the 1998 International Symposium on Underwater Technology. Japan, 370-374, (1998).

7. Ye Manzhu, Liao Shifang, Bao Fuhua. Application of 3D Modeling Technology in Cultural Relics Protection[J]. 2017.

8. Ju Junting, Zhong Gaojian, Yang Feiyun. Analysis based on reverse engineering measurement technology, "Modern Machinery"[M](2007).

9. Jin Tao, Product 3D Model Reconstruction Technology and Application Research in Reverse Engineering [J]. Zhejiang University. (2000).

10. Zhang Peichen. Application of Computer Aided Design (CAD) in the Restoration of Cultural Relics[J]. Cultural Relics Conservation and Archaeology, 2005 (02).

11. Xu Dan, on the effect of $3 \mathrm{D}$ scanning and printing technology on the protection of cultural relics[J].Shanghai University, School of Digital Arts.Shanghai, 2018.

12. Zhang Xuanru. Research and development of digital image restoration technology of cultural relics[M]. "Digital Design" 2017.

13. Yang Chenglei, Zhang Zongxia, Pan Rongjiang, Meng Xiangxu, Computer-aided cultural relic restoration system architecture and key technology research[J]. Journal of System Simulation.2006(07). 